\title{
The Impact of Incubators on Noise Transmission Produced by High-Frequency Oscillatory Ventilator Inside the Neonatal Intensive Care Unit
}

\author{
H. Salama*, A. Azzam, S. A. Omar, K. Abdulhadi \\ Women's Hospital, Department of pediatrics, Hamad Medical Corporation, BO box 3050
}

\begin{abstract}
Use of HFOV is a common practice inside the neonatal intensive care unit (NICU). There is a concern regarding the level of noise produced by HFOV inside the NICU. Objective: To evaluate and define the noise intensity produced by the high-frequency, oscillatory ventilator sensormedics (HFOV) and the impact of the incubator on the transmission of noise in different places inside the NICU. Methods: Using a noise analyzer, the noise produced by the HFOV sensormedics was measured in two setups: inside a quiet room, and inside the NICU during working hours. Measurements were repeated 2 times: first, inside and outside the tested incubator, second, inside and outside the next incubator. No human newborns were involved in the study; instead, a mannequin was used to simulate a newborn. The noise analyzer was placed next to mannequin mouth while inside the incubator and midway between the mannequin and the ventilator while measurements were taken outside the incubator. Each measurement was recorded against different HFOV $\boldsymbol{\Delta} \mathrm{P}$ and different frequency settings. Each measurement set lasted five minutes, where the highest and lowest readings were recorded every minute, and the average value of noise intensity was calculated.
\end{abstract}

Keywords Noise, NICU, HFOV, Incubator

\section{Introduction}

HFOV sensormedics 3100 is a legendary respirator and it formulates the vast majority of the evidence-based medicine addressing HFOV in newborn infants over the last 20 years. Despite the efficiency of this respirator in ventilating the lung it is clear to those physicians came across working with this machine that it produce significant noise that can be easily recognized when approach the intensive care room.

Noise is an unattractive and unfavorable sound. Sound is a vibration in a medium, which is usually air. The loudness of sound is measured in decibels (dB). Most of our knowledge about the damage to people from noise comes from studies of people with occupational exposure to sound. The standard for a workplace is to disallow more than eight hours of exposure to $90 \mathrm{~dB}$, four hours to $95 \mathrm{~dB}$, two hours to $100 \mathrm{~dB}$, and no exposure allowed to continuous noise above $115 \mathrm{~dB}$ or impulse noise above $140 \mathrm{~dB}$. For the protection of the public health, the US Environmental Protection Agency has proposed a DNL (Day Night average sound Level) of $55 \mathrm{~dB}$ during working hours, and $45 \mathrm{~dB}$ during sleeping hours in

* Corresponding author:

hus3038@yahoo.com (H. Salama)

Published online at http://journal.sapub.org/ajmms

Copyright (C) 2011 Scientific \& Academic Publishing. All Rights Reserved neighborhoods, and $45 \mathrm{~dB}$ in daytime, and $35 \mathrm{~dB}$ at night time in hospitals (1).

Noise may damage auditory functions for fetuses and newborns. Additionally, many pregnant women are exposed to noise in the workplace $(2,3,4)$, causing the American Academy of Pediatrics to issue a statement in 1997 which reviewed accumulated evidence that updated the affects of noise on pregnant women, since the previous report was published in 1974. This new report addressed the concern that fetuses and newborns exposed to excessive noise may suffer noise-induced hearing loss and other health effects $(5$, 6). In one study, Surenthiran et al demonstrated that the proximity of the post-nasal space to the inner ear is enough to allow noise to be transmitted, and may cause cochlear damage, and therefore hearing loss in infants receiving the higher flow rates CPAP (7). Furthermore, Berens et al addressed concerns about the level of the noise produced by HFOV equipment inside pediatric intensive care units (8). While using HFOV is common practice inside a NICU, and despite the high noise levels produced by the HFOV machine itself (9), there is little information available on the magnitude of the noise levels produced by the HFOV within different NICU setups.

\section{The Objective}


To describe the noise level produced by the HFOV sensormedics ${ }^{\mathrm{TM}}$ machine inside the neonatal intensive care environment and the effects of the incubator on reducing or augmenting the noise produced.

\section{Methods}

This is a non-clinical observational trial, conducted in February 2010, and lasted one month inside the neonatal intensive care unit of Women's Hospital, Hamad Medical Corporation in the State of Qatar. The intensive care unit consists of 28 intensive cots, located inside two large halls which are connected by a 3-meter-wide, imaginary door. The rest of the unit is composed of three separate, large halls that constituted intermediate care.

\section{Patients}

There were no human subjects involved in this trial. The measurements were conducted in one of the large halls, and no special arrangements for noise were taken during measurements inside the NICU. While special attention was taken inside the quiet room to limit the noise level during recording, the noise intensity and the test was performed after working hours.

\section{Equipments}

T HFOV Sensormedics 3100A Yorba, Linda California ${ }^{\mathrm{TM}}$. Incubators: Ohmeda Omnibed Giraffe ${ }^{\mathrm{TM}}$ double walled.

Noise analyzer: Monarch ${ }^{\mathrm{TM}}$ sound level meter.

The noise analyzer measured the noise intensity (loudness) at two different locations. Location (1) is inside one of the NICU large halls during the weekday (9-12am). Location (2) is inside a quiet room that was formerly the respiratory therapy equipment room when the test was conducted after working hours.

Noise measurements: Figure 1

A. Quiet Room: three sets of measurements were taken before and during the HFOV:

1. Measurement 1: Inside the incubator. A microphone was placed at the nearest point to the mouth of mannequin.

2. Measurement 2: Immediately outside the incubator adjacent to the HFOV

3. Measurement 3: Inside the neighboring incubator (2.5-meter distance).

B. Daytime NICU: three sets of measurements were taken before and during the HFOV was in operation:

1. Measurement 1: Inside the incubator. The microphone will be placed at the nearest point to the mouth of mannequin (Picture 3-a).

2. Measurement 2: Immediately outside the incubator, adjacent to the HFOV.
3. Measurement 3: Inside the neighboring incubators (2.5-meter distance).

C. HFOV Settings: The measurements were completed at different frequencies and amplitudes, with the highest amplitude (delta $\mathbf{\Delta} \mathrm{P}$ ) at 50, and the lowest frequency was 7.

D. Noise Analyzer: The analyzer measured both loudness (dB) and frequency of noise level.

E. Two persons were included in the measurements. One person was assigned to record the noise level and adjust the HFOV settings. The other person was assigned to observe and record the noise level using the noise analyzer.

F. Each Measurement lasted five minutes, and recorded the highest and lowest readings at one minute intervals.

G. The mean noise level was considered after five minutes of observation.

\section{Results}

Inside the tested incubator: The base line noise level before test start was $50 \mathrm{~dB}$ and $45 \mathrm{~dB}$ inside the tested incubator in both NICU and quite room respectively. The mean noise level recorded inside the NICU and the quiet room was $84( \pm 5) \mathrm{dB}$ and $78 \mathrm{~dB}( \pm 2)$ respectively. These levels were recorded inside the tested incubator, with HFOV settings at a frequency of $7 \mathrm{~Hz}$ and $\boldsymbol{\Delta} \mathrm{P}$ of 50 . The noise records were progressively reduced as HFOV $\boldsymbol{\Delta} \mathrm{P}$ decreased to reach to the lowest records (78dB inside NICU and $70 \mathrm{~dB}$ inside quite room), at $\boldsymbol{\Delta} \mathrm{P}$ of 20 and a frequency of 15 inside the NICU and inside the quiet room, respectively (Table 1) and graph 2

Outside the tested incubator: The mean measured noise level was $67( \pm 2) \mathrm{dB}$ in the quiet room, and $77 \mathrm{~dB}( \pm 5)$ inside the NICU during a normal working day.

Neighbor incubator: The mean records inside the incubator were $56 \mathrm{~dB}$ in a quiet room, and $63 \mathrm{~dB}$ inside the NICU. The mean records outside the incubator were $(63 \mathrm{~dB}(+-2)$ in a quiet room, and $63 \mathrm{~dB}( \pm 4)$ inside the NICU) (Tables 1 , Figure 2\&3).

\section{Conclusions}

The NICU is a place where humans and technology come together to produce a unique and sometimes unbearable level of noise that can cause a significant level of discomfort. Noise has been a major concern among neonatal environmentalists, who are concerned about the outcome of noise intensity in sick, newborn infants. One of the new advanced technologies introduced to neonatal practice is the HFOV of different types. In this observational, in-vitro study, we demonstrated that the noise produced by a popular HFOV machine (sensormedics) was considerably high enough to cause major concern $(86 \mathrm{~dB})$ inside the NICU.

Only two papers address noise produced by HFOV, and both were conducted in a non-NICU setup.

In this observation, we tried to document detailed information about the noise level at different settings. 
Table 1. Noise level recorded at different places. Records using different HFOV setting and data presented as mean value.

\begin{tabular}{|c|c|c|c|c|c|c|c|c|c|c|c|c|c|c|c|c|}
\hline \multirow{3}{*}{$\frac{\text { FRQ }}{\text { AMP }}$} & \multicolumn{4}{|c|}{ Noise inside incubator } & \multicolumn{4}{|c|}{ Outside incubator } & \multicolumn{4}{|c|}{ inside next incubator } & \multicolumn{4}{|c|}{$\begin{array}{l}\text { The farthest point out side incu- } \\
\text { bator }\end{array}$} \\
\hline & \multicolumn{2}{|c|}{15} & \multicolumn{2}{|c|}{7} & \multicolumn{2}{|c|}{15} & \multicolumn{2}{|r|}{7} & \multicolumn{2}{|c|}{15} & \multicolumn{2}{|c|}{7} & \multicolumn{2}{|c|}{15} & \multicolumn{2}{|c|}{7} \\
\hline & $\mathrm{Q}$ & NICU & $\mathrm{Q}$ & NICU & $\mathrm{Q}$ & NICU & $\mathrm{Q}$ & NICU & $\mathrm{Q}$ & NICU & $\mathrm{Q}$ & NICU & $\mathrm{Q}$ & NICU & $\mathrm{Q}$ & NICU \\
\hline 50 & 75 & 84 & 78 & 84 & 67 & 74 & 67 & 77 & 58 & 59 & 56 & 63 & 64 & 71 & 63 & 66 \\
\hline 45 & 75 & 76 & 78 & 79 & 64 & 75 & 64 & 71 & 57 & 61 & 54 & 63 & 62 & 74 & 61 & 66 \\
\hline 40 & 74 & 75 & 77 & 75 & 63 & 76 & 63 & 73 & 56 & 62 & 54 & 65 & 61 & 65 & 60 & 65 \\
\hline 35 & 73 & 75 & 76 & 75 & 63 & 75 & 62 & 74 & 55 & 61 & 53 & 63 & 60 & 67 & 58 & 63 \\
\hline 30 & 72 & 74 & 75 & 74 & 62 & 74 & 62 & 71 & 54 & 61 & 53 & 63 & 59 & 67 & 57 & 65 \\
\hline 25 & 70 & 73 & 73 & 74 & 62 & 74 & 62 & 71 & 53 & 60 & 52 & 63 & 58 & 66 & 57 & 63 \\
\hline 20 & 71 & 70 & 72 & 73 & 62 & 72 & 62 & 71 & 53 & 58 & 52 & 63 & 57 & 68 & 56 & 63 \\
\hline
\end{tabular}
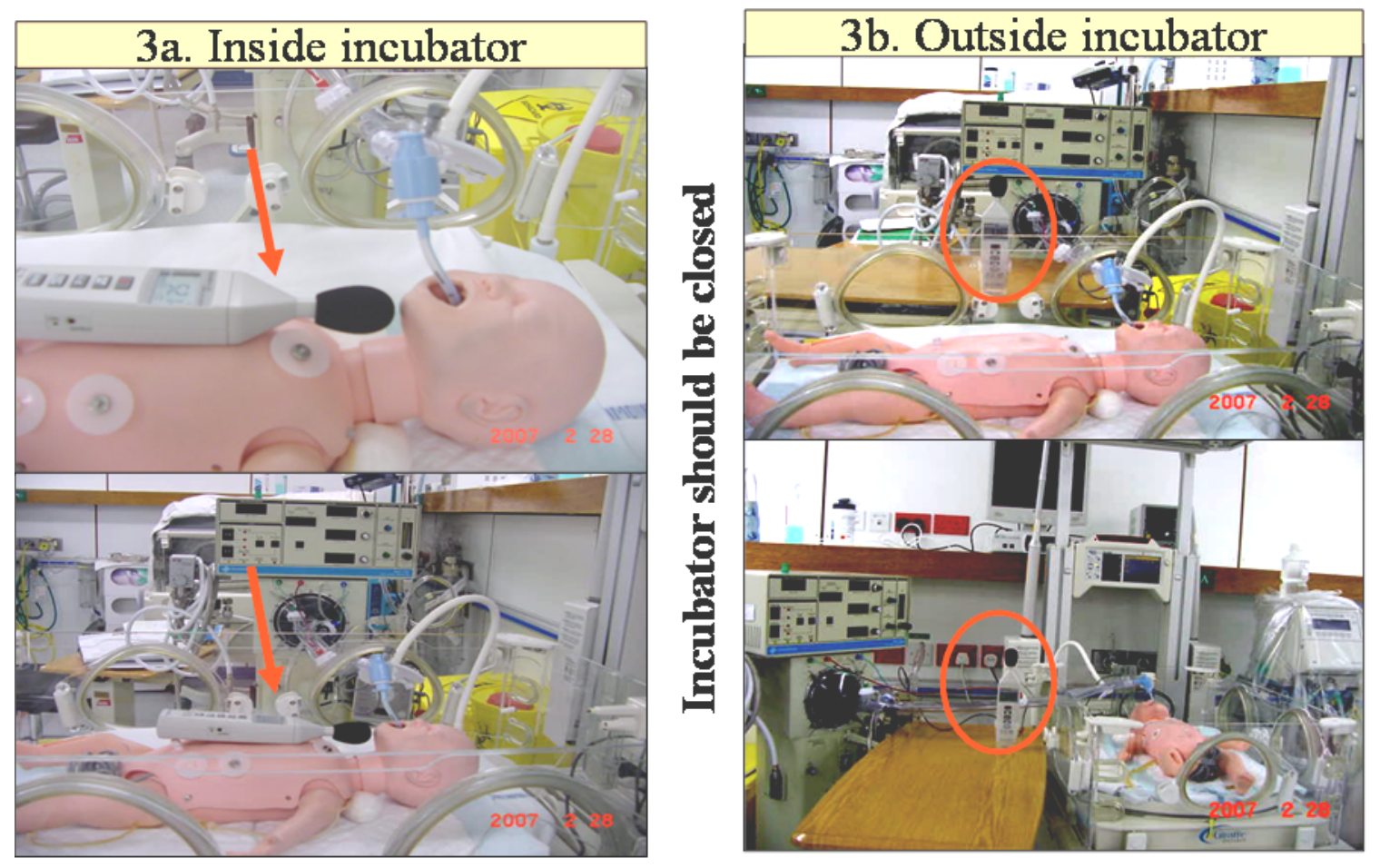

Figure 1. Measuring method of the noise.

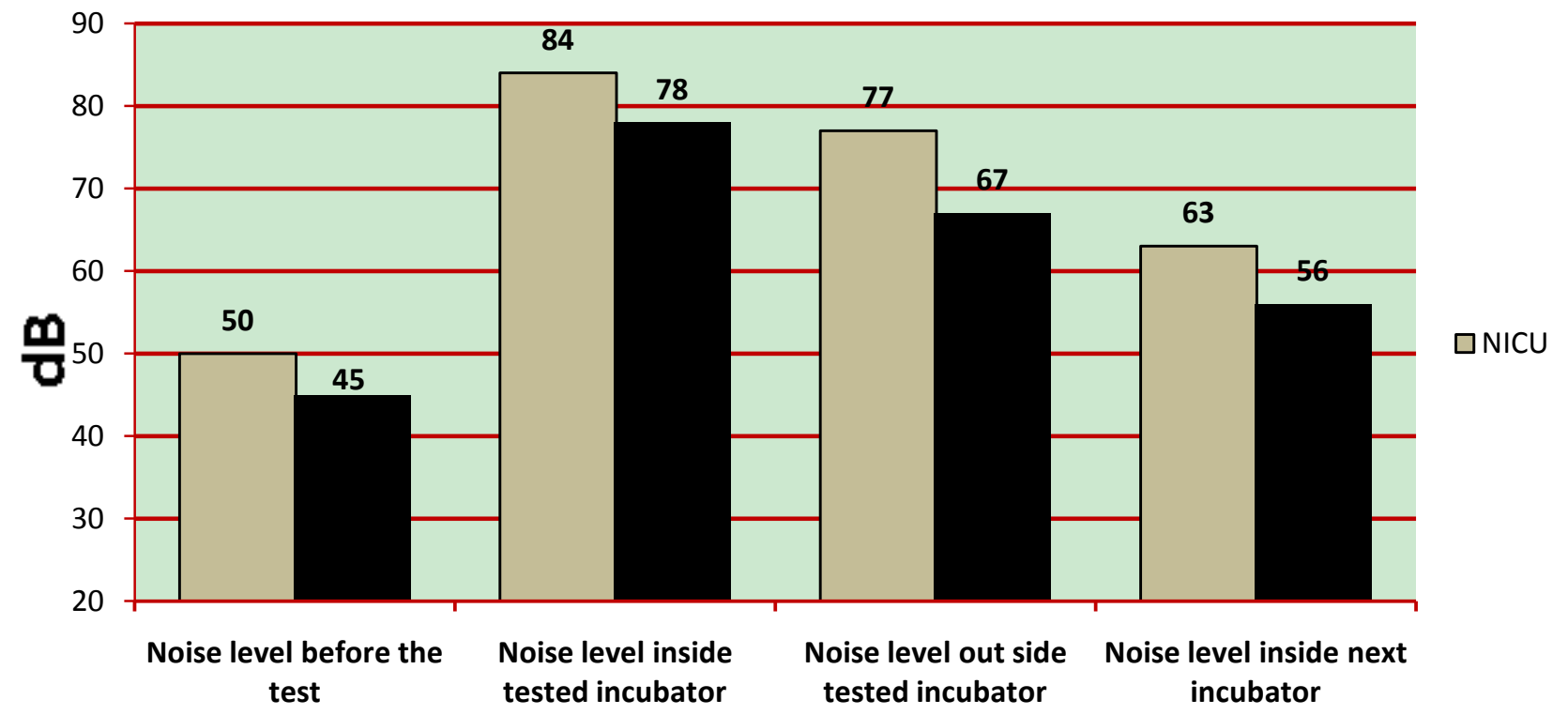

Figure 2. Comparing highest recorded noise level before the test and during the test. 


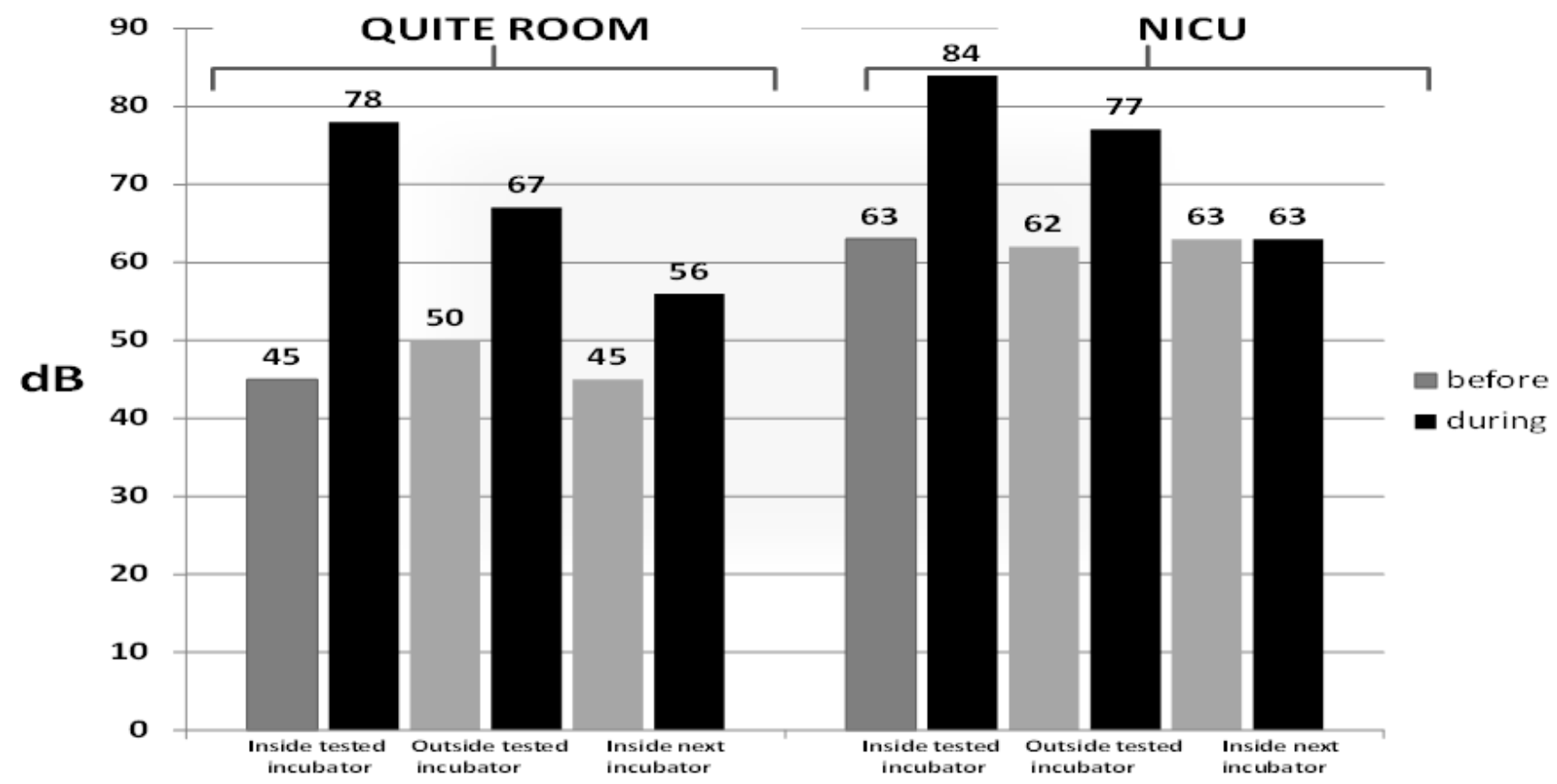

Figure 3. Overall highest recorded noise level in different locations before and during HFOV.

HFOV to determine a relation between noise intensity and variable levels of both amplitude and frequency of the HFOV. The noise records in different places inside the NICU demonstrate the significant effect of the incubator on the noise intensity transmitted, either through augmenting or reducing the noise level.

The fact that there was $40 \%$ rise in noise level produced by the HFOV machine may sound disturbing to several neonatal professions who are interested in a quite NICU environment. The incubators tested in this observation has a double wall, which clearly kept noise inside the tested incubator, and prevented noise from spreading outside the tested incubator, and reached up to $84 \mathrm{~dB}, 79 \mathrm{~dB}$ inside the tested incubator when the used amplitude was 7 . However, the reverse occurred in the neighbor incubator, where the noise transmitted inside the incubator was significantly less, and reached up to $56 \mathrm{~dB}$. This implies that the infant inside the neighboring incubator should be more protected from the noise. Another area of interest was that the highest noise level was recorded when HFOV $\boldsymbol{\Delta} \mathrm{P}$ was at its peak of 50 . Combining high amplitude with low frequency, however, did not add a significant difference in noise production (Table 1). There was little noise transmitted to the farthest point inside both the quiet room and NICU. The significant difference in noise recorded between the NICU and the quiet room stand for the noise inside the NICU, which is an added burden on ventilated infants.

Over the past two decades, the American Academy of Pediatrics has recommended that NICU noise amounts should not exceed 45 decibels $(\mathrm{dB})(5,6)$. More recently, new standards assert that the average noise levels inside NICU incubators should not exceed $60 \mathrm{~dB}(7,8)$. Subsequent work similarly recommended that NICU sound limitations of
50 to $55 \mathrm{~dB}$ on average, with a 1 -second peak of $70 \mathrm{~dB}$, should be implemented (5).

However, typical NICU noise frequently surpasses these recommended standards. Specifically, research indicates that normal NICU activity, including alarms sounding, incubator doors opening and closing, people tapping on incubators, and chairs scraping across floors, generates noise levels ranging from 65 to $90 \mathrm{~dB}$. Moreover, some researchers indicate that sound amounts in the NICU typically range from 45 to $135 \mathrm{~dB}$, occasionally averaging $80 \mathrm{~dB}$ for extended intervals of time, and frequently spiking to $117 \mathrm{~dB}(9,10$, and 11).

Numerous noise-measurement studies have been conducted in intensive care nurseries (12), and a few reports have measured sound frequencies of respiratory equipment (7, 8, and 9). Only two studies address the HFOV issue, but these are never measured inside NICU. These studies do not specify categories or correlate between different HFOV settings. In addition, these studies do not examine the effects of various distances from an HFOV machine, the relation to incubators, or consider neighboring incubators. In our study, we make theis data more clear, and this is an important reference when an HFOV of this kind is to used, and the noise transmission to both ventilated and neighboring infants is to be considered.

One report measured sound frequencies within the ear and intranasal while the infant received nasal CPAP versus conventional ventilation, or nasal cannula. This study was the first research to report sound spectral analysis (SSA) in a tertiary-level NICU. The results indicate that high-frequency sound is a significant problem in contemporary NICUs (7). The second study compares noise levels caused by four different, high-frequency ventilators, and sensormedics 
scored the highest in noise level inside the pediatric intensive care unit. Their work also documented the relationship between noise level and HFOV settings. They demonstrated that high $\mathbf{\Delta} \mathrm{P}$ will produce more noise, with the highest noise level at 70dB when HFOV $\boldsymbol{\Delta} \mathrm{P}$ was 70 . (9). This report documents that amplitude is the main, noise-producing setting irrespective of the HFOV frequency rate.

Our study could identify the effects of the incubator on augmenting the noise within the tested incubator and reducing the noise transmitted to the neighbor incubator. The hearing status of infants subjected to HFOV is not known, as no data available. Although Hosono et al compared the hearing of infants who received HFOV using a hummingbird oscillator with a control group of infants. The results were not significant in a small sample size of babies (15). It is sound logic to reserving using HFOV sensormedics machine to critical cases until more data is available about the hearing tests of these infants. Using earmuffs on these infants may reduce the risk of exposure to high noise levels produced by the machine.

The highest noise level was recorded inside the examined incubator. The highest noise level was noted when a high amplitude was applied. The lowest noise recorded inside the NICU was inside the neighboring incubator, which acts as a protecting shield.

HFOV sensormedics machine do produce significant noise level of significant concerns. The noise was augmented within the tested incubator while reduced inside the neighbor incubator. Given the data presented, it is recommended that noise protective tools should be used for infants receiving respiratory support using this machine as well as neighboring to infants who are using a HFOV. Based on this data, we implemented a developmental bundle of actions within our NICU that included; limiting use of sensormedics to cases where no response to other commercially available high frequency machines, such infants should wear earmuffs to reduce the level of noise, and that adjacent infants to those who are using an HFOV should be nursed inside incubators and also wear ear muffs.

\section{REFERENCES}

[1] Environmental Protection Agency, Office of Noise Abatement and Control. Information on levels of environmental noise requisite to protect Public Health and welfare With an Adequate Margin of Safety (Report No. 5509-74-004).
Washington, DC: Government Printing Office; 1974

[2] Kam PC, Kam AC, Thompson JF. Noise pollution in the anaesthetic and intensive care environment. Anaesthesia. 1994; 49:982-986

[3] Rudolph L, Forest CS. Female reproductive toxicology. In: LaDou J, ed. Occupational Medicine. Norwalk CT: Appleton \& Lange; 1990:275-287

[4] Paul Med. Occupational and Environmental Reproductive Hazards. A Guide for Clinicians. Baltimore. Williams \& Wilkins; 1993: xviii

[5] Noise: A hazard for the fetus and newborn committee on environmental health. American academy of pediatrics. Pediatrics vol. 100 No. 4 October 1997, pp. 724-727

[6] American academy of pediatrics, committee on environmental hazards noise pollution: neonatal aspects. Pediatrics. 1974; 54:476-479

[7] Surenthiran S S, Wilbraham K, May J, Chant T, Emmerson A J B, Newton V E. Noise levels within the ear and post-nasal space in neonates in intensive care. Archives of Disease in Childhood - Fetal- Neonata Edition 2003;88: F315-F318; doi:10.1136/fn.88.4.F315

[8] Berens R J. Noise in the pediatric intensive care unit. Journal of intensive care medicine vol 14 Issue 4, Pages 118-129

[9] Hoehn T, Busch A, Krause M. F. Comparison of noise levels caused by four different neonatal high-frequency ventilators. Intensive Care Med (2000) 26: 84 \pm 87

[10] Gray L, Philbin MK. Measuring sound in hospital nurseries. J of Perinatology, Dec 2000 (8 Pt 2), S100-4

[11] Brandon DH, Ryan DJ, Barnes AH. Effect of Environmental Changes on Noise in the NICU. Advances in Neonatal Care, Volume 8 Number 5, supplement: October 2008 Pages S5-S10

[12] Byers J, Lowman L., Waugh R Neonatal intensive care unit sound levels environment, and infant response, Neonatal Intensive Care 18 (2005), pp. 48-53

[13] Kellam B, Bhatia J. Sound spectral analysis in the neonatal intensive care unit: Measuring high frequency sound, Journal of Pediatric Nursing 23 (2008), pp. 317-323

[14] Bamiou FE, Luxon L.M. Aetiology and clinical presentations of auditory processing disorders - a review, Archives of Disease in Childhood 85 (2001), pp. 361-365

[15] Hosono S, Imura S, Akiyama K, Ttakigawa I, Shezeki S, Takeda $\mathrm{H}$. Effect of vibration on auditory organ systems in low-birth weight infants treated with high-frequency oscillatory ventilation: 5 year outcome. Pediatrics International (2001) 43, 16-19 